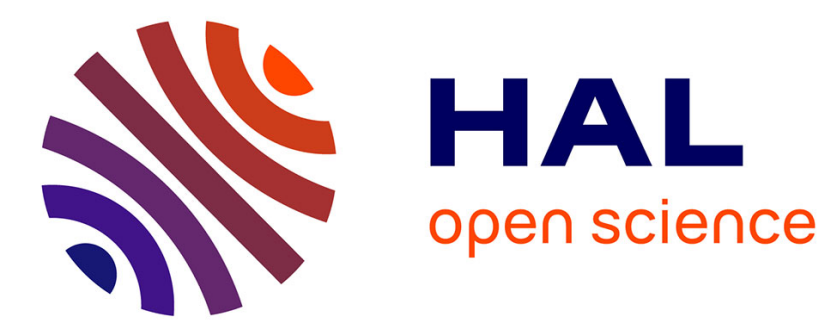

\title{
Pour une nouvelle approche de la motivation entrepreneuriale
}

Inès Gabarret, Benjamin Vedel

\section{To cite this version:}

Inès Gabarret, Benjamin Vedel. Pour une nouvelle approche de la motivation entrepreneuriale. La Revue des Sciences de Gestion, 2015, 1 (271), pp.13-20. 10.3917/rsg.271.0013 . hal-01891934

\section{HAL Id: hal-01891934 \\ https://hal.science/hal-01891934}

Submitted on 10 Oct 2018

HAL is a multi-disciplinary open access archive for the deposit and dissemination of scientific research documents, whether they are published or not. The documents may come from teaching and research institutions in France or abroad, or from public or private research centers.
L'archive ouverte pluridisciplinaire HAL, est destinée au dépôt et à la diffusion de documents scientifiques de niveau recherche, publiés ou non, émanant des établissements d'enseignement et de recherche français ou étrangers, des laboratoires publics ou privés. 


\title{
Pour une nouvelle approche de la motivation entrepreneuriale
}

\author{
Par Inès Gabarret et Benjamin Vedel
}

\author{
Inès GABARRET \\ Professeur Associé \\ EDC Paris Business School \\ Paris, France
}

OCRE - LABEX Entreprendre

\section{Benjamin VEDEL}

Maître de Conférences

IAE Lille

Lille, France

LEM-CNRS (UMR 9221) - LABEX Entreprendre

\section{Résumé}

La littérature sur la motivation entrepreneuriale considère que la création d'une entreprise peut être choisie ou subie. Ainsi, elle est majoritairement étudiée suivant la dichotomie push/pull ou nécessité/opportunité. Dans cet article, nous partons d'un questionnement à propos de la capacité explicative d'une approche binaire de la motivation entrepreneuriale et proposons une adaptation, en enlevant l'exclusivité du choix. La motivation entrepreneuriale est ainsi étudiée au travers de deux dimensions : économique et non-économique et interprétée à la lumière des aspects positifs ou négatifs, issus de la personnalité de l'entrepreneur (optimisme/pessimisme).

Mots-clés : motivation entrepreneuriale, push-pull, opportunité-nécessité 


\begin{abstract}
The literature on entrepreneurial motivation considers the creation of a business as chosen or endured. Thus, it is mostly studied following the push/pull or necessity/opportunity dichotomies. This article questions the explanatory capacity of a binary approach of entrepreneurial motivation and proposes an adaptation, by removing the exclusivity of choice. Entrepreneurial motivation is then studied through two dimensions: economic and noneconomic and interpreted in the light of positive or negative aspects, linked to the entrepreneur's personality (optimism /pessimism).
\end{abstract}

Key words : entrepreneurial motivation, push-pull, opportunity-necessity

\title{
Resumen
}

La literatura en motivacion empresarial considera que la creacion de empresa puede ser una eleccion o una necesidad. De esta manera, la motivacion se estudia a traves de las dicotomias push/pull o necesidad/oportunidad. Este artículo cuestiona la capacidad explicativa de un enfoque binario de la motivación empresarial y propone una adaptación, mediante la eliminación de la exclusividad de la elección. La motivación emprendedora sera estudiada a través de dos dimensiones: económica y no económica e interpretada a la luz de aspectos positivos o negativos, vinculados a la personalidad del empresario (optimismo/pesimismo).

Palabras clave : motivacion empresaria, push-pull, oportunidad-necesidad 


\section{Pour une nouvelle approche de la motivation entrepreneuriale}

La motivation est un sujet connu de la littérature de gestion et a longtemps été étudiée en dehors du cadre de l'entrepreneuriat. Elle a été initialement définie comme l'énergie qui pousse et conduit l'action (P. Olson et D. Bosserman, 1984). En effet, un premier groupe d'auteurs considère la motivation comme une impulsion qui vise, soit la satisfaction d'un besoin (A. Maslow, 1954 ; F. Herzberg, 1959 ; C. Alderfer, 1969 ; D. McClelland, 1953), soit l'atteinte d'objectifs fixés à l'avance (E. Locke, 1968). D'autres auteurs expliquent la motivation comme une réponse attitudinale vis-à-vis d'une situation qui semble injuste (J. Adams, 1963) ou qui permet la réalisation d'une attente (V. Vroom, 1964). D'un point de vue psychologique, la motivation a été expliquée comme un conditionnement, un comportement qui viserait l'évitement de la douleur et la recherche du bonheur et du plaisir (B. F. Skinner, 1963). Enfin, une dernière grille de lecture divise la motivation en deux sousdimensions : intrinsèque et extrinsèque. La première fait référence à la recherche d'un intérêt personnel, tandis que la deuxième regarde la récompense qui suit un comportement déterminé, tel que l'argent et le statut (A. Carsrud et M. Brannback, 2011).

Dans ce texte, nous nous intéressons à la motivation entrepreneuriale. La littérature sur la motivation entrepreneuriale considère que la création d'une entreprise peut être choisie ou subie. Elle peut être déclenchée par la présence d'une opportunité d'affaires, ou par un besoin de rémunération en cas de chômage. Suivant cette optique, deux dimensions exclusives apparaissent habituellement, appelées respectivement «push » et « pull ».

L'approche « push » correspond au concept d'entrepreneuriat par nécessité (Z. Acs et al. 2005; P. Reynolds et al. 2002). Il comporte deux aspects. Le premier, de nature économique, concerne le fait de ne pas avoir d'emploi. Le deuxième, de nature non économique, est matérialisé par l'insatisfaction au travail (D. Bradley et J. Roberts, 2005; C. Stoner et F. Fry, 1982; R. Brockhaus, 1980; A. Cooper, 1971). Dans l'autre extrême, l'approche «pull » correspond à l'entrepreneur par opportunité. Il comporte aussi deux volets (économique et non 
économique) : la découverte d'une opportunité d'affaires et le désir d'indépendance (Z. Acs et al. 2005; P. Reynolds et al. 2002). Une particularité de cette approche est le caractère mutuellement exclusif des dimensions «push/pull».

Or, de nos jours, les mutations de l'environnement de travail et l'essor de l'entrepreneuriat, entre autres, ont donné naissance à des profils atypiques d'entrepreneurs. Toutes ces nouvelles figures d'entrepreneur se différencient de la vision monolithique de l'entrepreneur d'autrefois (A. Tounes et A. Fayolle, 2006). L'époque est à l'autonomie, à la recherche de sens (E.-M. Hernandez, 2006b). La question qui se pose alors est : dans quelle mesure l'approche nécessité/ opportunité est-elle adéquate pour interpréter la motivation entrepreneuriale ?

En effet, des études ont montré que la motivation entrepreneuriale pouvait varier avec le temps (N. Williams et C. Williams, 2012), et s'avérer différente dans chaque groupe d'entrepreneurs (par exemple dans le cas d'entrepreneurs de différentes origines ethniques comme l'expliquent A. Shapero et L. Sokol, 1982).

Ainsi, dans cet article, nous avançons que la multiplicité des situations de créations entrepreneuriales et de profils d'entrepreneur est imparfaitement retranscrite par les théories existantes et demande un éclairage nouveau.

Pour cela, nous avons, dans un premier temps, visé à déconstruire la motivation entrepreneuriale en enlevant le caractère d'exclusivité des concepts «pull» (opportunité, indépendance) et "push» (nécessité et insatisfaction). Quatre dimensions distinctes ont été observées : l'opportunité économique (représentée par la possibilité d'une augmentation de revenu), la nécessité (conséquence de l'absence d'emploi), l'insatisfaction avec le travail précédent, et les désirs d'autonomie et d'indépendance.

En parallèle, il apparaît que les analyses basées sur la distinction «push/pull » provoquent des difficultés au niveau de l'interprétation. En effet, le désir d'indépendance attire aussi bien qu'il pousse l'individu vers l'entrepreneuriat (K. Hughes, 2003). Nous soulignons dans un deuxième temps que les quatre dimensions peuvent être regardées d'une manière positive ou négative. Par exemple, la satisfaction au travail peut se comprendre comme un facteur négatif (l'insatisfaction qui pousse l'individu vers la création d'entreprise), ou comme un facteur positif (la recherche de satisfaction qui attire vers la création d'entreprise). 
Le plan de l'article est le suivant : dans une première partie, nous présenterons une revue de littérature sur la motivation entrepreneuriale, principalement le modèle «push/pull». Une deuxième partie sera dédiée à la compréhension des limites de l'approche étudiée et présentera une adaptation du modèle. Nous conclurons avec les implications théoriques et managériales.

\section{La motivation entrepreneuriale : une revue de littérature}

La motivation entrepreneuriale a été étudiée selon différentes approches. A. Carsrud et M. Brannback (2011) suggèrent la séparation entre les études économiques (suivant les écrits des économistes tels que J. A. Schumpeter, 1934 ; I. Kirzner, 1973, etc.), et psychologiques (par exemple dans le cas des entrepreneurs sociaux et des «lifestyle entrepreneurs »). Pour sa part, S. Shane et al. (2003) proposent de classer les théories de la motivation en deux groupes : (1) les théories générales qui s'intéressent aux besoins d'accomplissement, de contrôle, à la vision, au désir d'indépendance et à la passion de l'entrepreneur, et (2) les théories relatives à la tâche qui s'intéressent à la fixation d'objectifs ou au sentiment d'auto-efficacité.

Une grande partie de la littérature économique en entrepreneuriat considère que la décision de créer une entreprise est le résultat d'un processus de maximisation, dans lequel l'individu va comparer les revenus de différentes activités et va choisir celle qui va lui fournir le plus haut retour (P. Arenius et M. Minniti, 2005). Ainsi, les théories contemporaines de l'action expliquent le choix entrepreneuriat/salariat comme le résultat d'un calcul économique de probabilité (F. Facchini, 2007). Cette approche économique se retrouve dans une importante quantité d'écrits sur la motivation entrepreneuriale.

Comme nous l'avons indiqué dans l'introduction, la recherche sur la motivation entrepreneuriale est généralement divisée en deux corps principaux nommés «push » et « pull (J. Kirkwood et C. Cambell-Hunt, 2007 ; L. Schjoedt et K. Shaver, 2007; B. Gilad et P. Levine, 1986). Cette approche a donné naissance à deux types de motivation : celle par opportunité, dans le cas de la théorie «pull», et celle par nécessité, dans le cas de la théorie « push » (Z. Acs et al. 2005; P. Reynolds et al. 2002). Le Global Entrepreneurship Monitor (GEM), un programme qui décrit et analyse l'activité entrepreneuriale à travers le monde (Z. Acs et al. 2005), respecte cette double typologie. 


\subsection{La théorie pull : opportunité et indépendance}

La théorie « pull » voit la création d'entreprise comme le résultat d'une culture entrepreneuriale (K. Hughes, 2003). Elle serait motivée par une recherche d'indépendance et d'autonomie, mais aussi par le fait d'avoir repéré une opportunité d'affaires à développer.

Les deux dimensions qui constituent l'approche «pull» (l'opportunité d'affaires et le désir d'indépendance), sont souvent regardées de manière positive car elles considèrent la création d'entreprise comme un choix individuel et volontaire (J. Kirkwood et C. Campbell-Hunt, 2007). S. Shane et S. Venkataraman (2000) expliquent que la reconnaissance et l'exploitation d'opportunités d'affaires est un trait fondamental de l'entrepreneur. Pour ces auteurs, l'entrepreneuriat se présente comme un état transitoire, une réponse d'un individu face à une opportunité. Cette réponse peut se matérialiser aussi bien dans le cadre d'un contrat de travail que par le biais de la création d'entreprise nouvelle. La capacité à identifier des opportunités, en plus d'être la principale caractéristique entrepreneuriale (S. Shane et S. Venkataraman, 2000), est fortement corrélée à la décision de commencer une affaire (N. Langowitz et M. Minniti, 2007; P. Arenius et M. Minniti, 2005).

L'opportunité se définit comme une situation dans laquelle de nouvelles marchandises, matières premières, méthodes d'organisation et de nouveaux services peuvent être vendus à un prix plus important que leur coût de production (S. Shane et S. Venkataraman, 2000). Elle est satisfaisante si le bénéfice généré est supérieur au coût d'opportunité de l'entrepreneur (S. Shane et al. 2003).

Différents types d'opportunité peuvent être distingués suivant les écrits des économistes. Par exemple, pour J.A. Schumpeter (1934), l'opportunité se trouve dans l'innovation. L'entrepreneur est un innovateur qui introduit sur le marché de nouveaux produits qui vont remplacer les anciens (destruction créative). I. Kirzner (1973), de son côté, voit l'entrepreneur comme un arbitragiste qui va profiter des imperfections du marché pour trouver son bénéfice et, de cette manière, restituer l'équilibre. Les approches des deux économistes, d'après J. De Jong et O. Marsili (2011) sont complémentaires.

Le concept d'opportunité d'affaires a donné lieu à une littérature importante sans que sa définition ne fasse l'objet d'un consensus (M. Casson et N. Wadeson 2007). Au-delà des 
définitions Schumpétérienne ou Kirznerienne, elle a été aussi étudiée en tant que facteur de motivation «pull» (voir Z. Acs et al. 2005 ; P. Reynolds et al. 2002 ; J. Mc Mullen et al. 2008 ; J. Hessels et al. 2008).

D'autres auteurs se sont intéressés au concept d'opportunité en tant que processus : le passage de la découverte à l'exploitation, la construction d'opportunités, la transformation d'une idée en opportunité d'affaires, etc. (voir W. Gartner et al. 1992 ; A. Shapero et L. Sokol, 1982). Finalement, une vaste littérature met en rapport l'opportunité et les traits de personnalité. Le fait de posséder certains traits de personnalité permettrait la perception des opportunités d'affaires (I. Grilo et R. Thurik, 2004). Il apparait que certains individus ont la capacité de percevoir des opportunités depuis leur très jeune âge. Des fois, le fait d'appartenir à une famille d'entrepreneurs semblerait aider dans le développement de ces caractéristiques (B. Gilad et P. Levine, 1986).

Le concept d'opportunité est présent dans la plupart des définitions de l'entrepreneuriat (S. Shane et S. Venkataraman, 2000 ; F. Adaman et P. Devine, 2002). Il peut se comprendre, finalement, comme une possibilité d'amélioration de revenus (S. Shane et al. 2003). Ainsi, l'entrepreneur opportuniste, comme décrit par P. Reynolds et al. (2002), est conduit principalement par le désir et la recherche d'un gain économique (A. Carsrud et M. Brännback, 2011).

Cependant, au-delà des questions économiques, certains entrepreneurs peuvent être attirés vers la création d'entreprise par un désir d'indépendance, même si le bénéfice est limité (K. Hughes, 2003). L'hypothèse d'une motivation non économique est aussi suggérée par J. Hessels et al. (2008). Dans leur étude, des entrepreneurs indépendants ne semblent pas avoir été motivés par la recherche d'une augmentation de richesse, mais plutôt par le fait d'avoir un niveau de vie confortable. Aussi E.-M. Hernandez (2006a), dans son observation de quarante entrepreneurs français, confirme que la principale motivation du créateur est son désir d'autonomie. D. Bradley et J. Roberts (2004) s'accordent sur l'idée que des individus peuvent créer des entreprises basées sur le rejet des emplois routiniers et ennuyeux. Ces entrepreneurs, n'étant pas motivés par une augmentation de revenus, recherchent de l'autonomie et des nouveaux défis. 
S. Cromie (1987) trouve que les désirs d'autonomie, d'accomplissement et de satisfaction au travail, poussent les individus vers l'entrepreneuriat, d'une manière plus importante que les facteurs de motivation économique.

Ainsi, dans le concept «pull » de la motivation, deux dimensions se voient regroupées : (1) la recherche d'une amélioration économique, généralement interprétée comme opportunité, et (2) un désir d'indépendance et d'autonomie. Or, suivant le résultat de plusieurs recherches, (K. Hughes, 2003 ; J. Hessels et al. 2008), ces deux concepts ne semblent pas apparaître systématiquement de manière conjointe.

\subsection{La théorie push : nécessité et insatisfaction}

Deux dimensions viennent constituer l'approche «push » de la motivation entrepreneuriale : (1) la nécessité (absence d'une autre possibilité d'emploi, chômage prolongé, pas d'employabilité), et (2) l'insatisfaction (le fait d'avoir un travail insatisfaisant).

Les facteurs de poussée sont souvent accompagnés de connotations négatives (J. Kirkwood et C. Campbell-Hunt, 2007) parce qu'ils représentent, entre autres, la nécessité, l'absence de travail, le licenciement, l'emploi précaire ou flexible, la restructuration d'entreprise (J. Mc Mullen et al. 2008 ; K. Hughes, 2003).

Les entrepreneurs motivés par des facteurs push peuvent être considérés comme des individus rejetés par la société qui cherchent à prouver leur valeur à travers la création d'entreprises (B. Gilad et P. Levine, 1986). Les individus peuvent se voir également poussés vers l'entrepreneuriat par des situations conflictuelles comme l'insatisfaction dans leur travail précédent (D. Bradley et J. Roberts, 2005; C. Stoner et F. Fry, 1982; R. Brockhaus, 1980; A. Cooper, 1971).

Pourtant, l'insatisfaction au travail n'est pas une notion propre à l'entrepreneuriat (R. Brockhaus, 1980). Dans les études en Ressources Humaines, le «turnover », ou rotation du personnel, s'explique généralement comme un manque d'implication dû, entre autres, à une insatisfaction au travail. Cette insatisfaction peut pousser l'individu vers un changement de poste ou d'organisation, comme le montre l'étude de C. Besseyre des Horts et V. Nguyen (2010), dans lequel l'insatisfaction au travail se présente comme le déterminant principal de l'intention de départ. 
La question qui se pose, alors, est de savoir pourquoi certains individus salariés créent de nouvelles sociétés au lieu de rechercher un autre emploi. Selon R. Brockhaus (1980), la décision de créer une nouvelle activité, au lieu de chercher un poste, est liée à l'intensité de l'insatisfaction. D'après l'auteur, il est possible que des entrepreneurs auraient été si peu satisfaits avec l'emploi précédent, qu'ils auraient considéré peu probable de trouver un emploi satisfaisant dans une autre organisation (R. Brockhaus, 1980). L'intensité de l'insatisfaction a aussi été étudiée par C. Stoner et F. Fry (1982). Ils ont suggéré une relation entre l'intensité de l'insatisfaction dans l'emploi précédent et le type d'affaire ou d'industrie choisie pour le projet de création. Une forte insatisfaction semble mener les individus vers un changement d'industrie ou de secteur d'activité.

Ainsi, dans l'approche push, comme dans l'approche pull, nous nous trouvons face à deux facteurs (insatisfaction et nécessité) qui peuvent ne pas apparaître simultanément.

\subsection{D'autres facteurs de motivation}

Même s'il existe une importante littérature à propos de la motivation «push/pull », d'autres recherches montrent que le besoin d'accomplissement, la possibilité de créer son propre emploi et le désir d'autonomie, se placent, souvent, au premier rang des citations de la motivation des créateurs (T. Verstraete et B. Saporta, 2006). De plus en plus d'études indiquent que la motivation est difficilement représentée par des catégories exclusives, surtout dès que l'on considère de nouveaux profils d'entrepreneurs. En effet, dans l'étude de J. Vik et G. McElwee (2011) sur les agriculteurs entrepreneurs norvégiens, les motivations sociales sont au même niveau d'importance que les motivations économiques. Pour sa part, E.-M. Hernandez (2006b), explique que dans la société postmoderne, atomisée et individualiste, les individus trouvent de plus en plus dans la création d'entreprise une possibilité de donner du sens à leur vie. Ainsi,

parmi les lifestyle entrepreneurs, et dans le cas de l'entrepreneuriat social ou familial, il existe, habituellement, une claire orientation non économique de la motivation. Pour les lifestyle entrepreneurs, la création d'entreprise est considérée comme une manière d'améliorer leur qualité de vie (M. Peters et al. 2009). Pour les entrepreneurs sociaux, c'est la recherche d'une amélioration pour la société, d'un gain social (A. Carsrud et M. Brannback, 2011). En général, il est considéré que le déclencheur de la motivation, dans ce cas-là, est la constatation d'un 
besoin qui concerne un groupe social ou professionnel, une communauté ou un territoire (A. Tounes et A. Fayolle, 2006).

Les «SeniorPreneurs» se trouvent parmi les nouvelles catégories d'entrepreneurs. Ils se définissent comme étant des créateurs de valeurs économiques et sociales. Ainsi, d'après I. Safraou et al. (2012), leurs motivations se situent notamment dans les plans sociaux et durables.

La tendance non économique apparaît également dans les études sur les chercheurs entrepreneurs (généralement dans le cas des spin-offs). Ici, la connaissance scientifique est la dimension principale de la motivation, suivie de près par le besoin d'accomplissement et le désir d'indépendance (S. Morales Gualdron et al. 2009).

Toutefois, dans tous les cas cités ci-dessus, le choix de création d'entreprise se montre comme une manière de subvenir aux besoins en faisant une activité qui apporte de la satisfaction. Pour les chercheurs entrepreneurs, c'est leur désir de transmission ou d'exploitation des connaissances scientifiques. Pour les entrepreneurs sociaux, c'est leur volonté de contribuer à l'amélioration de l'environnement ou de la société. Finalement, pour les lifestyle entrepreneurs, leur satisfaction est atteinte grâce à une meilleure qualité de vie.

La littérature précise que les individus deviennent des entrepreneurs pour des raisons multiples, et que des motifs non économiques rivalisent fortement avec les motifs économiques (S. Cromie, 1987).

\section{Les limites du modèle : Pour une nouvelle approche de la motivation entrepreneuriale}

Au-delà des multiples approches pour étudier la motivation entrepreneuriale, le modèle «push/pull » (qui est à la base de l'approche motivationnelle nécessité/opportunité) reste le plus utilisé dans les recherches en Sciences de Gestion (N. Williams et C. Williams, 2012). La facilité d'utilisation du modèle a fait sa réussite. Le modèle a fait ses preuves et est applicable à l'échelle mondiale (E. Eijdenberg et E. Masurel, 2013). Pourtant, l'outil est critiqué par rapport à l'exclusivité du choix (E. Eijdenberg et E. Masurel, 2013 ; N. Williams et C. Williams, 2012 ; I. Verheul et al. 2010 ; J. Kirkwood, 2009; K. Hughes, 2003 ; L. Stevenson, 1990) et nécessite 
une adaptation. Le modèle, basé sur le point de vue des économistes, est restrictif quand il s'agit d'expliquer les motivations des nouveaux profils d'entrepreneurs. Par la suite, nous allons discuter des limites du modèle et proposer une adaptation permettant de les dépasser.

\subsection{Le caractère exclusif du modèle nécessité/opportunité et l’impossibilité de représenter la motivation non économique}

Une des caractéristiques principales de l'approche «push/pull» concerne l'exclusivité du choix. Cet aspect peut mener à une mauvaise interprétation de la motivation. En effet, les études du GEM montrent que les individus enquêtés sont forcés de choisir entre une motivation « push » ou « pull», n'ayant pas d'espace pour une option entre ces deux extrêmes. Ainsi, comme le reconnaît le GEM dans son rapport 2008 (N. Bosma et al. 2009), ceux qui se trouvent au milieu, ont tendance à choisir la dernière option. Cela s'explique par les connotations négatives associées à l'entrepreneuriat par nécessité (J. Kirkwood et C. Campbell-Hunt, 2007). Les conclusions de l'étude GEM peuvent ainsi sous-estimer l'entrepreneuriat par nécessité et surestimer celui par opportunité (N. Bosma et al. 2009).

En général, la création d'entreprise est considérée comme déclenchée davantage par des situations négatives (comme la guerre, le chômage, l'insatisfaction) que par des situations positives (A. Shapero et L. Sokol, 1982). Or, dans la plupart des cas, les changements de vie d'un individu seront provoqués par une combinaison de facteurs positifs et négatifs (A. Shapero et L. Sokol, 1982), c'est-à-dire par des combinaisons de facteurs de poussée et de traction. Face aux problèmes d'une classification mutuellement exclusive, plusieurs auteurs proposent que la motivation entrepreneuriale soit étudiée comme un continuum le long duquel différentes combinaisons de facteurs puissent exister (E. Eijdenberg et E. Masurel, 2013 ; N. Williams et C. Williams, 2012 ; I. Verheul et al. 2010 ; J. Kirkwood, 2009; K. Hughes, 2003 ; L. Stevenson, 1990).

Figure 1 : La motivation push et pull 


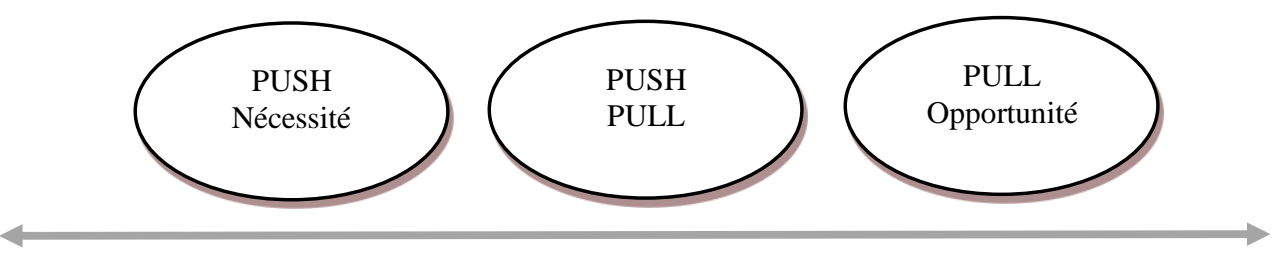

Ainsi, les dernières études en motivation entrepreneuriale classifient les entrepreneurs en trois groupes : motivation «push», motivation «pull» et motivations mélangées («mixed motivation ») (I. Verheul et al. 2010). Cependant, le fait d'admettre que les entrepreneurs puissent être motivés par des compositions de facteurs ne nous renseigne en rien sur leur nature. Pour aller plus loin dans la compréhension de la composition de la motivation, nous avons besoin d'un outil qui nous permette d'identifier les facteurs qui sont regroupés dans cette motivation mélangée.

D'autre part, le fait de regrouper des motivations économiques et non économiques dans chaque dimension ne permet pas de choisir des facteurs non économiques de façon isolée. Par exemple, un entrepreneur lifestyle qui est en recherche d'une qualité de vie (motivation non économique) ne pourra pas signifier sa préférence au travers de l'approche nécessité/opportunité. Sa motivation, qui correspond à une recherche de satisfaction dans la relation travail/qualité de vie (motivation «pull » non économique) n'est pas représentée dans le modèle.

\subsection{La faiblesse de l'opposition dans l'approche « push/pull »}

La classification de la motivation en «push » et «pull » provoque, aussi, des problèmes dans l'interprétation des résultats de la recherche. K. Hughes (2003) montre que, certaines variables citées par les entrepreneurs comme des variables de motivation «push» (le désir d'indépendance qui va attirer l'individu vers l'entrepreneuriat) peuvent aussi être interprétées comme étant des motivations de nécessité «push » (le manque d'indépendance dans le travail précédent). Cela provoque des contradictions dans les études scientifiques. Le désir d'être son propre patron, d'être autonome, est classé parmi les facteurs «pull» par l'étude GEM. Toutefois, d'autres auteurs le considèrent parmi les facteurs « push » de la motivation (comme par exemple dans le texte d'O. Giacomin et al. 2008). 
Concernant l'exemple déjà cité de l'entrepreneur lifestyle qui cherche la satisfaction dans la relation travail/qualité de vie, sa motivation («pull» et non économique) ne peut pas être représentée par la motivation «push » d'insatisfaction au travail.

\subsection{Une adaptation de l'outil pour comprendre la motivation entrepreneuriale}

Dans cet article, nous avons vu que la motivation entrepreneuriale se définissait, en général, au travers de deux catégories exclusives.

Tableau 1 : Les catégories exclusives de la motivation entrepreneuriale

\begin{tabular}{|c|c|}
\hline Push & Pull \\
\hline Nécessité / Insatisfaction & Opportunité / Indépendance \\
\hline
\end{tabular}

Source : Gabarret et Vedel (2012)

Deux problèmes ont été associés à cette classification. D'une part, il s'agit du manque de représentativité d'un choix exclusif. Une solution à cela est proposée par certains auteurs, celle de comprendre la motivation comme un continuum «push/pull » (I. Verheul et al. 2010 ; J. Kirkwood, 2009; K. Hughes, 2003 ; L. Stevenson, 1990). D’autre part, nous nous trouvons face au caractère ambigu de la classification «push/pull». Un même concept peut être interprété aussi bien comme «push » ou «pull» (K. Hughes, 2003).

La motivation entrepreneuriale est donc un sujet complexe (J. Kirkwood et C. Cambell-Hunt, 2007), composée de multiples facteurs, parmi lesquels sont fréquemment cités : le désir d'indépendance, l'appât du gain, le besoin d'accomplissement, la personnalité de l'individu, l'identification d'une opportunité, la recherche d'un style de vie, le contrôle de sa propre destinée, la sécurité économique pour sa famille, la construction d'une affaire transmissible, etc. (T. Verstraete, 1999).

Au-delà de cette complexité, certains éléments apparaissent régulièrement (Y. Robichaud et al., 2001 ; D. Kuratko et al. 1997) : (1) L'importance d'une récompense extrinsèque monétaire (l'acquisition de richesses, l'augmentation du revenu); (2) Le besoin d'une récompense 
intrinsèque liée à l'accomplissement d'une tâche (poursuivre un défi, se développer) ; (3) La recherche d'autonomie et d'indépendance (conserver sa liberté, créer son propre emploi, être son propre chef, contrôler sa destinée); et (4) La sécurité de sa famille (assurer le futur économique de sa famille, créer une affaire pour transmettre à ses enfants).

E-M. Hernandez (2006a), pour sa part, à partir d'une étude empirique sur 40 entrepreneurs, retient aussi quatre types de motivation entrepreneuriale : (1) passion/réalisation personnelle, (2) autonomie, (3) mieux gagner sa vie/faire fortune, et (4) obtention d'un revenu. Ces résultats peuvent se rapprocher des résultats des recherches préalables. Une cinquième dimension avait été évoquée par l'auteur (puissance/besoin de pouvoir) mais elle n'est finalement pas soutenue par son analyse.

En croisant la littérature précédemment citée, différentes propositions peuvent être formulées afin d'élargir la compréhension et l'étude de la motivation entrepreneuriale. La première, est une possible classification de la motivation entrepreneuriale en deux groupes de facteurs : économiques et non économiques (tableau 2).

\section{Tableau 2 : Les dimensions de la motivation entrepreneuriale dans la littérature}

\begin{tabular}{|c|l|l|l|}
\hline \multirow{5}{*}{ ECONOMIQUE } & $\begin{array}{l}\text { Z. Acs et al. (2005) } \\
\text { P. Reynolds et al. } \\
\text { (2002) }\end{array}$ & $\begin{array}{l}\text { Y. Robichaud et al. } \\
\text { (2001) } \\
\text { D. Kuratko et al. } \\
(1997)\end{array}$ & $\begin{array}{l}\text { E-M. Hernandez } \\
\text { (2006a) }\end{array}$ \\
\cline { 2 - 5 } & Pull/Opportunité & $\begin{array}{c}\text { Extrinsèque } \\
\text { monétaire }\end{array}$ & $\begin{array}{c}\text { Mieux gagner sa } \\
\text { vie/Faire fortune }\end{array}$ \\
\hline \multirow{2}{*}{$\begin{array}{c}\text { NON } \\
\text { ECONOMIQUE }\end{array}$} & Pull/Indépendance & \begin{tabular}{c} 
Sécurité \\
économique \\
Indépendance \\
\cline { 2 - 5 }
\end{tabular} & $\begin{array}{c}\text { Obtention d'un } \\
\text { revenu }\end{array}$ \\
\cline { 2 - 5 } & Push/Insatisfaction & $\begin{array}{c}\text { Intrinsèque } \\
\text { développement }\end{array}$ & $\begin{array}{c}\text { Passion/Réalisation } \\
\text { personnelle }\end{array}$ \\
\hline
\end{tabular}

Il apparaît, aussi, qu'au-delà des classifications «push/pull », la motivation entrepreneuriale peut se comprendre au travers de quatre grandes composantes principales, dont deux à caractère économique : (1) le besoin d'un moyen de subsistance ou la création de son propre emploi face à un manque de possibilités sur le marché du travail, et (2) l'envie d'améliorer ses revenus ou la possibilité d'exploiter une affaire hautement rentable. Deux autres composantes sont définies 
comme non économiques : (1) la recherche d'un travail satisfaisant, dans lequel l'individu peut exprimer toutes ses capacités et réaliser tous ses rêves professionnels, et (2) le désir d'indépendance et d'autonomie, de n'avoir à rendre des comptes qu'à soi-même.

\section{Figure 2 : Les composantes de la motivation entrepreneuriale}

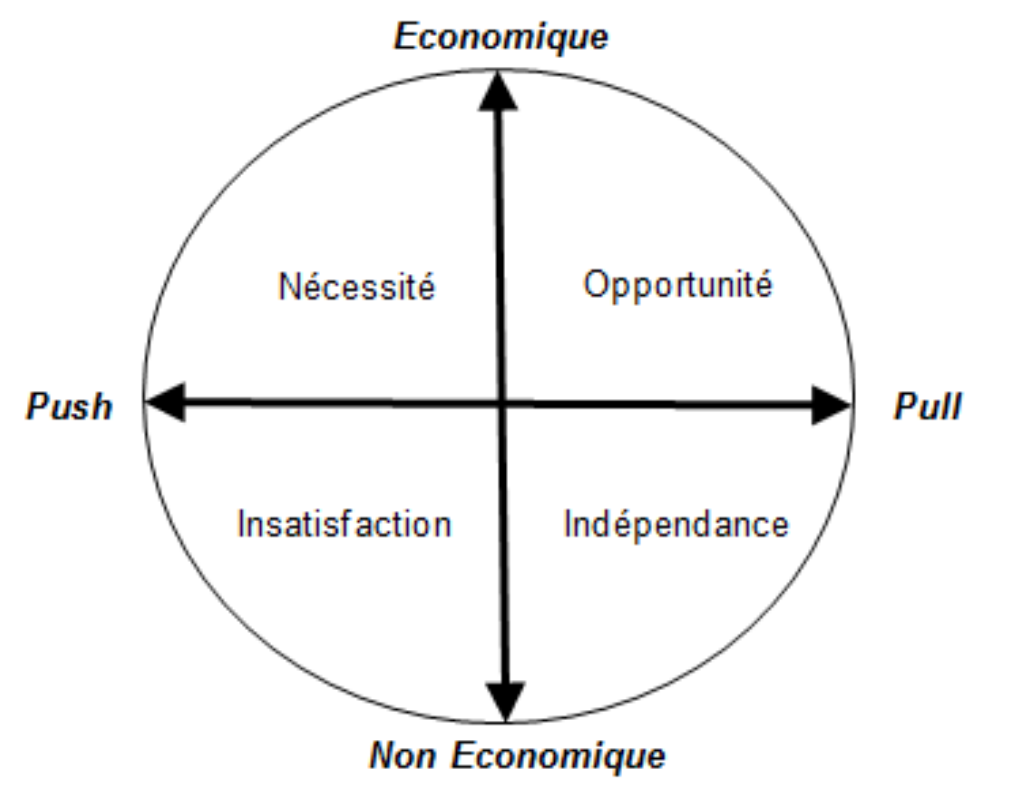

Source : Gabarret et Vedel (2012)

Cette représentation en quatre composantes permet de comprendre la motivation entrepreneuriale comme des combinaisons particulières de différents facteurs. Un individu peut se sentir motivé par la création entrepreneuriale parce qu'il a trouvé une opportunité d'affaires (motivation par opportunité), mais, ce même individu peut se sentir insatisfait dans son travail et vouloir quitter son poste (motivation par insatisfaction). Dans cet exemple, et dans le cadre des enquêtes de motivation entrepreneuriale du GEM, l'individu se voit limité à choisir une motivation «pull » (opportunité) ou «push » (insatisfaction). Soit l'un, soit l'autre. Son choix ne sera pas complètement représentatif de sa motivation, car elle est, en réalité, une composition de facteurs «pull » et «push » (figure 3, cas 1). Aussi, un individu peut se sentir insatisfait dans son travail (motivation par insatisfaction) mais ne pas vouloir trouver un autre emploi, attiré par un désir d'autonomie et d'indépendance (motivation par indépendance). Ici, leur choix 
(«push»ou «pull») sera aussi partiellement représentatif (figure 3, cas 2). En enlevant l'exclusivité dans le choix des facteurs motivationnels, les chercheurs peuvent évaluer les raisons qui conduisent chaque individu vers la création entrepreneuriale et présenter un tableau plus représentatif de leurs réelles motivations.

\section{Figure 3 : La motivation entrepreneuriale par regroupement de facteurs individuels}

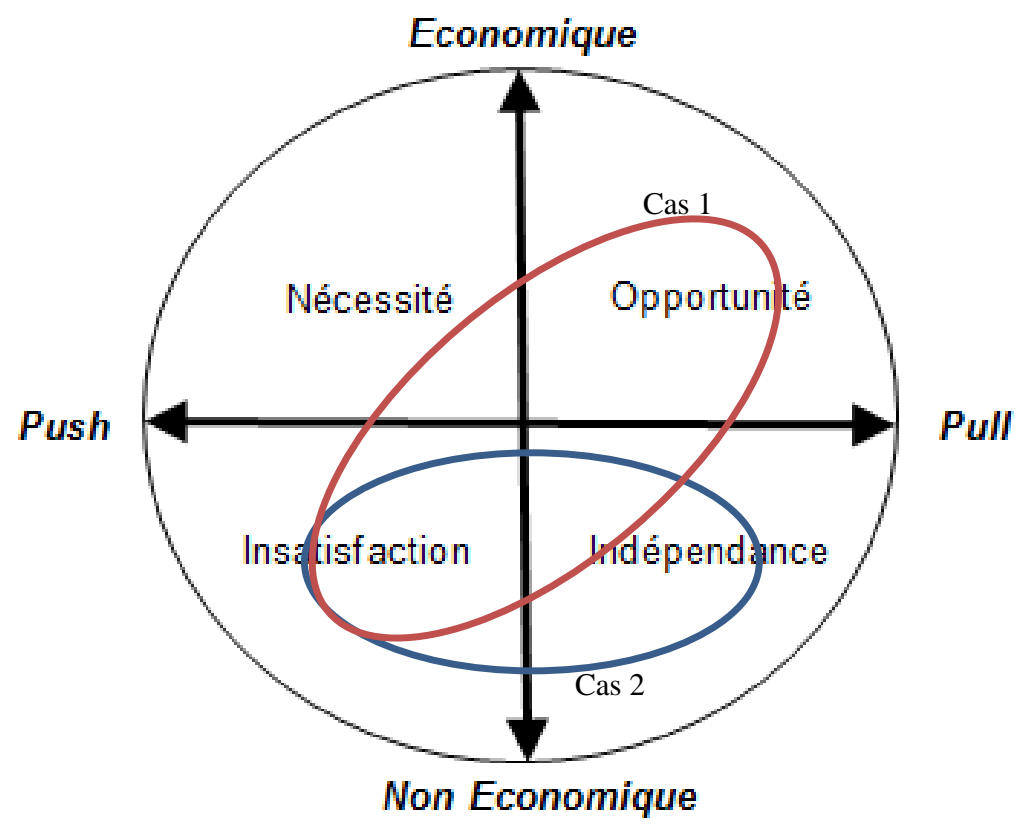

Cependant, comme nous l'avons déjà montré, la classification «push/pull » reste ambigüe. Une manière d'enlever cette ambivalence est d'interpréter les facteurs depuis une optique différente. Nous proposons que toutes les dimensions puissent être interprétées soit comme «push », soit comme « pull ». Cela dépendra de la situation particulière de chaque entrepreneur et de la façon dans laquelle il veut se représenter. L'indépendance, l'argent et l'emploi peuvent s'interpréter comme des manques ou des désirs. Concernant l'insatisfaction au travail, F. Herzberg (1959) souligne que la recherche de satisfaction est indépendante de l'insatisfaction au travail. Ainsi, si d'un côté, nous avons de l'insatisfaction ou un manque de satisfaction au travail, de l'autre, nous avons une recherche de satisfaction.

Tableau 3 : Les dimensions de la motivation entrepreneuriale 


\begin{tabular}{|c|l|l|}
\cline { 2 - 3 } \multicolumn{1}{c|}{} & \multicolumn{1}{c|}{ Négatif } & \multicolumn{1}{c|}{ Positif } \\
\hline Non-économique & $\begin{array}{l}\text { Manque de satisfaction } \\
\text { Manque d'autonomie }\end{array}$ & $\begin{array}{l}\text { Désir de satisfaction } \\
\text { Désir d'indépendance }\end{array}$ \\
\hline Economique & $\begin{array}{l}\text { Manque d'emploi } \\
\text { Manque d'argent }\end{array}$ & $\begin{array}{l}\text { Désir de travailler } \\
\text { Désir d'augmentation de } \\
\text { revenu }\end{array}$ \\
\hline
\end{tabular}

Les individus se différencient les uns des autres par leurs caractéristiques de personnalité, leurs désirs, leurs attentes, etc. Par exemple, un individu qui aimerait gagner plus d'argent trouvera sa motivation plutôt parmi les facteurs économiques, tandis qu'un individu de personnalité optimiste trouvera sa motivation préférentiellement parmi les facteurs positifs. Il est à croire que le choix des facteurs motivationnels dépendra, entre autres, des caractéristiques de personnalité de l'individu, de ses désirs et attentes, et des possibilités perçues d'opportunités. Il devient alors possible d'interpréter la motivation entrepreneuriale au regard de ces quatre dimensions laissant aux individus le choix de l'assemblage des facteurs de motivation qui leur correspondent le plus (Figure 4).

Figure 4 : La motivation entrepreneuriale au travers des deux dimensions E/NE - P/N 


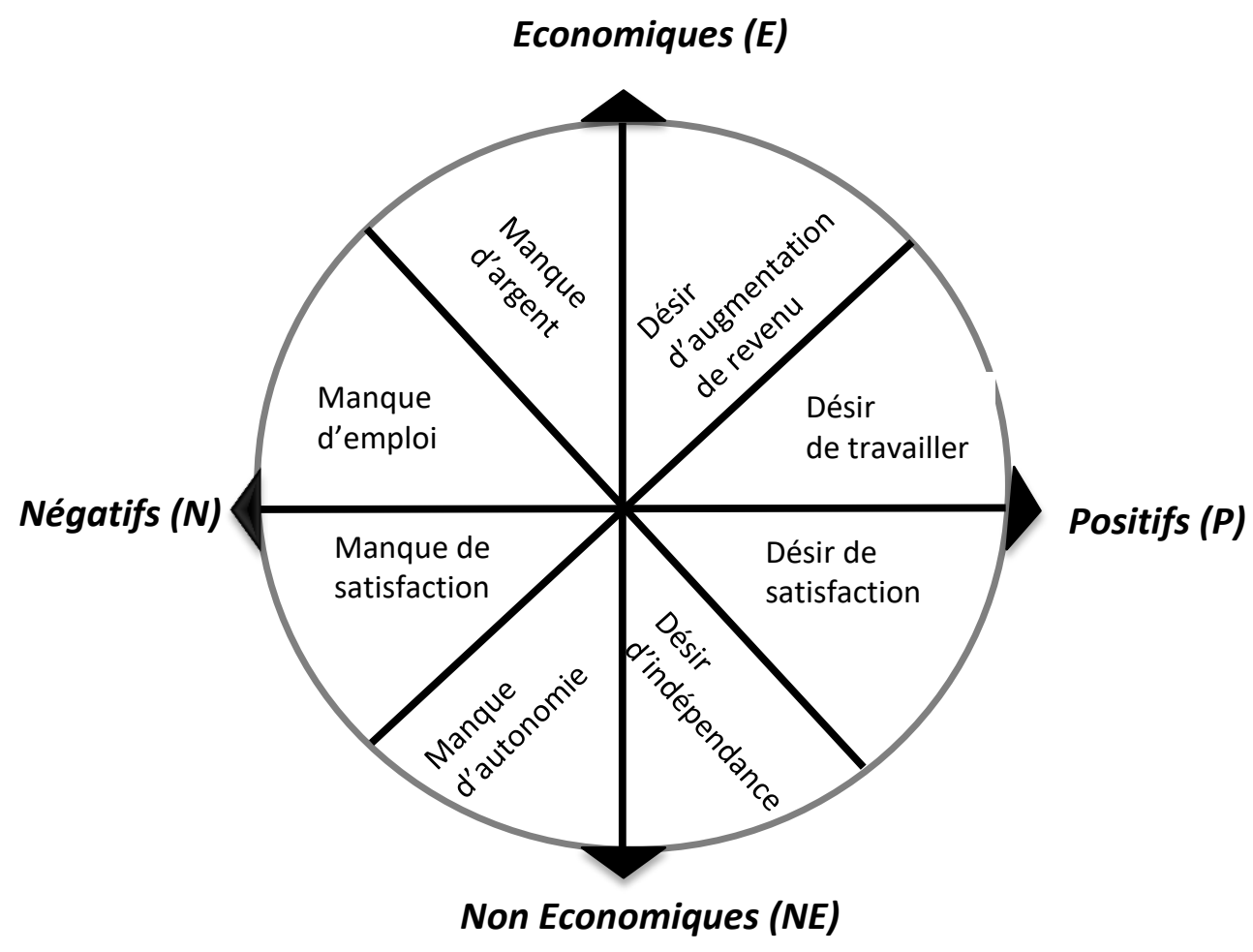

\section{Conclusion}

L'entrepreneuriat s'impose de plus en plus, se développe et se complexifie. L'économie postmoderne devient une économie d'entrepreneurs, dans laquelle la création d'entreprise joue un rôle primordial, aussi bien économique que social (A. Tounes et A. Fayolle, 2006).

La motivation entrepreneuriale est un concept qui a été très étudié, mais qui est encore susceptible d'évoluer, face à l'essor de l'entrepreneuriat et à l'entrée dans le champ de la création d'entreprise de nouveaux profils d'entrepreneurs. Cette étude a cherché à contribuer à sa compréhension.

Dans ce texte, nous avons effectué, à partir d'une large revue de la littérature, un travail de déconstruction du concept de la motivation entrepreneuriale. Suite à cela, un nouveau regard sur la motivation entrepreneuriale a été formulé, permettant de mettre en place la base pour de futures recherches. Cette manière de recomposer la motivation à partir de multiples 
combinaisons de facteurs doit permettre, selon nous, d'atteindre une plus grande représentativité des situations observées.

La motivation entrepreneuriale est aussi un puissant déterminant de la création d'entreprise. Il permet d'expliquer et de catégoriser les types de création, ainsi que la survie des entreprises créées (Y. Robichaud et al. 2001). De plus, certaines études indiquent l'existence d'un lien entre motivation et performance (M. Vivarelli, 2004 ; J. Block et M. Wagner, 2010). Ceci implique que des aides (accompagnement, financement) puissent être accordées plus facilement à un type d'entrepreneur au détriment d'un autre (J. Block et M. Wagner, 2010).

Notre étude donne des clefs de compréhension de la motivation entrepreneuriale afin de permettre aux organismes institutionnels, associations locales et nationales, ainsi qu'aux acteurs du secteur privé, intéressés par la création d'entreprise, de surveiller et d'analyser la motivation entrepreneuriale de manière plus fine. Finalement, le fait de comprendre les mobiles qui animent les individus pour créer des entreprises, permettra aussi de proposer un accompagnement personnalisé adéquat. 


\section{Références bibliographiques}

Acs Z., Arenius P., Hay M., Minniti M. (2005), Global Entrepreneurship Monitor, 2004 Executive Report, Babson College and London Business School.

Adaman F., Devine P. (2002), "A Reconsideration of the Theory of Entrepreneurship: a participatory approach", Review of Political Economy, Vol. 14, n 3, p. 329-355.

Adams J. (1963), “Towards an Understanding of Inequality”, Journal of Abnormal and Normal Social Psychology, Vol. 67, p. 422-436.

Alderfer C. (1969), “An Empirical Test of a New Theory of Human Need”, Organizational Behavior \& Human Performance, Vol. 4, $\mathrm{n}^{\circ}$ 2, p. 142-175.

Arenius P., Minniti M. (2005), "Perceptual Variables and Nascent Entrepreneurship", Small Business Economics, Vol. 24, p. 233-247.

Besseyre des Horts C., Nguyen V. (2010), «Satisfaction, implication, engagement, enracinement et intention de départ des jeunes cadres: Une relation ambigüe », Congrès AGRH, 17-19 novembre, Rennes, Saint-Malo, France.

Block J., Wagner M. (2010), "Necessity and Opportunity Entrepreneurs in Germany: Characteristics and Earnings Differentials", Schmalenbach Business Review, Vol. 62, p. 154-174.

Bosma N., Acs Z., Autio E., Coduras A., Levie J. (2009), Global Entrepreneurship Monitor, 2008 Executive Report, Babson College and Universidad del Desarrollo.

Bradley D., Roberts J. (2004), "Self-Employment and Job Satisfaction: Investigating the Role of SelfEfficacy, Depression, and Seniority”, Journal of Small Business Management, Vol. 42, Issue 1, p. 37.

Bradley D., Roberts J. (2005), "Is Low Job Satisfaction a Cause of Self-Employment Entrance? A Survival Analysis Using Data from the British Household Panel Survey", working paper, East Carolina University, Greenville, North Carolina, 27 Mai.

Brockhaus R. (1980), "The Effect of Job Dissatisfaction on the Decision to Start a Business", Journal of Small Business Management, Vol. 18, p. 37-43.

Carsrud A., Brannback M. (2011), "Entrepreneurial Motivations: What Do We Still Need to Know?" Journal of Small Business Management, Vol. 49, $\mathrm{n}^{\circ}$ 1, p. 9-26.

Casson M., Wadeson N. (2007), "The Discovery of Opportunities: Extending the Economic Theory of the Entrepreneur", Small Business Economics, Vol. 28, p. 285-300.

Cooper A. (1971). The Founding of Technologically-Based Firms. The Center For Venture Management, Milwaukee, Wisconsin.

Cromie S. (1987), "Motivations of Aspiring Male and Female Entrepreneurs", Journal of Occupational Behavior, Vol. 8, $\mathrm{n}^{\circ}$ 3, p. 251-261.

De Jong J., Marsili O. (2011), Schumpeterian and Kirznerian opportunities: An empirical investigation of opportunity types, DRUID, Copenhagen Business School, Denmark, June 15-17.

Eijdenberg E., Masurel E. (2013), "Entrepreneurial Motivation in a Least Developed Country: Push Factors and Pull Factors among Mses in Uganda", Journal of Enterprising Culture, Vol. 21, n 1, p. 1943. 
Facchini F. (2007), «L'entrepreneur comme un homme prudent », La Revue des Sciences de Gestion, $\mathrm{n}^{\circ} 226-227$, p. 29-38.

Gabarret I., Vedel B. (2012), «Quitter son emploi pour devenir entrepreneur », @grh, n4 4, p.79-97.

Gartner W., Bird B., Starr J. (1992), “Acting As If: Differentiating Entrepreneurial from Organizational behavior", Entrepreneurship Theory and Practice, spring, p. 13-31.

Giacomin O., Guyot J. L., Janssen F. (2008), “The Influence of Necessity and Opportunity Motivations on the Choice of an Economic Sector: an Empirical Study of Belgian Entrepreneurs", XXII Research in Entrepreneurship and Small Business conference (RENT XXII), November, Covilha, Portugal.

Gilad B., Levine P. (1986), “A Behavioral Model of Entrepreneurial Supply”, Journal of Small Business Management, October, p. 45-53.

Grilo I., Thurik R. (2004), "Determinants of Entrepreneurship in Europe”, Papers on Entrepreneurship, Growth and Public Policy 2004-30, Max Planck Institute of Economics, Entrepreneurship, Growth and Public Policy Group, July.

Herzberg F. (1959). The Motivation to Work, New York, John Wiley \& Sons.

Hernandez E.-M. (2006a), « Les trois dimensions de la décision d'entreprendre », Revue française de gestion, $\mathrm{n}^{\circ} 168-169$, p. 337-357.

Hernandez E.-M. (2006b), «Extension du domaine de l'entrepreneur... et limites », La Revue des Sciences de Gestion, ${ }^{\circ} 219$, p. 17-26.

Hessels J., Van Gelderen M., Thurik R. (2008), "Entrepreneurial Aspirations, Motivations, and their Drivers", Small Business Economics, Vol. 31, p. 323-339.

Hughes K. (2003), “Pushed or Pulled? Women's Entry into Self-Employment and Small Business Ownership", Gender, Work and Organization, Vol. 10, n 4, p. 433-454.

Kirkwood J. (2009), "Motivational Factors in a Push-Pull Theory of Entrepreneurship", Gender in Management: An International Journal, Vol. 2, n 5, p. 346-364.

Kirkwood J., Campbell-Hunt C. (2007), "Using Multiple Paradigm Research Methodologies to Gain New Insights into Entrepreneurial Motivations", Journal of Enterprising Culture, Vol. 15, n 3, p. 219241.

Kirzner I. (1973), Competition and Entrepreneurship, Chicago, IL, US, University of Chicago Press.

Kuratko D., Hornsby J., Naffizger D. (1997), “An Examination of Owner's Goals in Sustaining Entrepreneurship", Journal of Small Business Management, January, p. 24-33.

Langowitz N., Minniti M. (2007), "The Entrepreneurial Propensity of Women", Entrepreneurship Theory and Practice, May, p. 341-364.

Locke E. (1968), "Toward a Theory of Task Motivation and Incentives", Organizational Behavior \& Human Performance, Vol. 3, $\mathrm{n}^{\circ}$ 2, p. 157-189.

Maslow A. (1954), Motivation and Personality, Harper \& Row Publishers Inc.

McClelland D. (1953). The Achievement Motive, New York, Appleton Century Crofts.

McMullen J., BAGBY D., PALICH L. (2008), "Economic Freedom and the Motivation to Engage in Entrepreneurial Action”, Entrepreneurship Theory and Practice, September, p. 875-895. 
Morales Gualdron S., Gutierrez Gracia A., Roig Dobon S. (2009), "The Entrepreneurial Motivation in Academia: a Multidimensional Construct", International Entrepreneurship and Management Journal, Vol. 5, n 3, p. 301-317.

Olson P., Bosserman D. (1984), “Attributes of the Entrepreneurial Type”, Business Horizons, Mai-June, p. 53-56.

Peters M., Frehse J., Buhalis D. (2009), “The Importance of Lifestyle Entrepreneurship: A Conceptual Study of the Tourism Industry", PASOS, Revista de Turismo y Patrimonio Cultural, Vol. 7, n ${ }^{\circ}$ 2, p. 393-405.

Reynolds P., Bygrave W., Autio E., Cox L., Hay M. (2002), "Global Entrepreneurship Monitor, 2002 Executive Report", Babson College, Ewing Marion Kauffman Foundation, and London Business School.

Robichaud Y., Mc Graw E., Roger A. (2001), “Toward the Development of a Measuring Instrument for Entrepreneurial Motivation", Journal of Developmental Entrepreneurship, Aug., Vol. 6, n² 2, p. 189201.

Safraou I., Castellano S., Maalaoui A., Menvielle L. (2012), « Singularité du processus entrepreneurial chez les seniors. Un entrepreneuriat social et solidaire », La Revue des Sciences de Gestion, ${ }^{\circ}{ }^{255-256,}$ p. 59-66.

Schjoedt L., Shaver K. (2007), "Deciding on an Entrepreneurial Career: A Test of the Pull and Push Hypotheses Using the Panel Study of Entrepreneurial Dynamics Data", Entrepreneurship Theory and Practice, September, p. 733-752.

Schumpeter J. A. (1934), The Theory of Economic Development, Cambridge, MA, US, Harvard University Press.

Shane S., Venkataraman S. (2000), "The Promise of Entrepreneurship as a Field of Research", The Academy of Management Review, Vol. 25, $\mathrm{n}^{\circ}$ 1, p. 217-226.

Shane S., Locke E., Collins C. (2003), "Entrepreneurial Motivation”, Human Resource Management Review, Vol. 13, p. 257-279.

Shapero A., Sokol L. (1982), "The Social Dimensions of Entrepreneurship”, in Kent, C.; Sexton, D.; and Vesper, K. (Ed.), Encyclopedia of Entrepreneurship, Prentice-Hall, Englewood Cliffs, New Jersey, p. $72-90$.

Skinner B. F. (1963),’Operant Behavior”, American Psychologist, Vol. 18, n 8, p. 503-515.

Stevenson L. (1990), "Some Methodological Problems Associated with Researching Women Entrepreneurs", Journal of Business Ethics, Vol. 9, p. 439-446.

Stoner C., Fry F. (1982), “The Entrepreneurial Decision: Dissatisfaction or Opportunity?” Journal of Small Business Management, Vol. 20, p. 39-44.

Tounes A., Fayolle A. (2006), «L'odyssée d'un concept et les multiples figures de l'entrepreneur », $L a$ Revue des Sciences de Gestion, ${ }^{\circ}$ 220-221, p. 17-30.

Verheul I., Thurik R., Hessels J., Van Der Zwan P. (2010), "Factors Influencing the Entrepreneurial Engagement of Opportunity and Necessity Entrepreneurs", SCALES, Scientific Analysis of Entrepreneurship and SMEs, march. 
Verstraete T. (1999), Entrepreneuriat. Connaître l'entrepreneur, comprendre ses actes, Editions L'Harmattan.

Verstraete T., Saporta B. (2006), Création d'entreprise et entrepreneuriat, Les Editions de l'ADREG.

Vik J., McElwee G. (2011), "Diversification and the Entrepreneurial Motivations of Farmers in Norway", Journal of Small Business Management, Vol. 49, n 3, p. 390-410.

Vivarelli M. (2004), “Are All the Potential Entrepreneurs So Good?” Small Business Economics, Vol. 23, p. 41-49.

Vroom V. (1964), Work and Motivation, New York, Wiley.

Williams N., Williams C. (2012), "Evaluating the socio-spatial contingency of entrepreneurial motivations: A case study of English deprived urban neighbourhoods", Entrepreneurship \& Regional Development, Vol. 24, n 7-8, p. 661-684. 